07,19

\title{
First-principles investigation of mechanical and thermodynamic properties of nickel silicides at finite temperature*
}

\author{
(C) Zhiqin Wen, Yuhong Zhao, Hua Hou, Liwen Chen \\ School of Materials Science and Engineering, North University of China, \\ Taiyuan, China \\ E-mail: zhaoyuhong@nuc.edu.cn
}

(Received 19 October 2017)

First-principles calculations are performed to investigate lattice parameters, elastic constants and 3D directional Young's modulus $E$ of nickel silicides (i. e. $\beta-\mathrm{Ni}_{3} \mathrm{Si}, \delta-\mathrm{Ni}_{2} \mathrm{Si}, \theta-\mathrm{Ni}_{2} \mathrm{Si}, \varepsilon-\mathrm{NiSi}$, and $\alpha-\mathrm{NiSi}_{2}$ ), and thermodynamic properties, such as the Debye temperature, heat capacity, volumetric thermal expansion coefficient, at finite temperature are also explored in combination with the quasi-harmonic Debye model. The calculated results are in a good agreement with available experimental and theoretical values. The five compounds demonstrate elastic anisotropy. The dependence on the direction of stiffness is the greatest for $\delta-\mathrm{Ni}_{2} \mathrm{Si}$ and $\theta-\mathrm{Ni}_{2} \mathrm{Si}$, when the stress is applied, while that for $\beta-\mathrm{Ni}_{3} \mathrm{Si}$ is minimal. The bulk modulus $B$ reduces with increasing temperature, implying that the resistance to volume deformation will weaken with temperature, and the capacity gradually descend for the compound sequence of $\beta-\mathrm{Ni}_{3} \mathrm{Si}>\delta-\mathrm{Ni}_{2} \mathrm{Si}>\theta-\mathrm{Ni}_{2} \mathrm{Si}>\varepsilon-\mathrm{NiSi}>\alpha-\mathrm{NiSi}_{2}$. The temperature dependence of the Debye temperature $\Theta_{D}$ is related to the change of lattice parameters, and $\Theta_{D}$ gradually decreases for the compound sequence of $\varepsilon-\mathrm{NiSi}>\beta-\mathrm{Ni}_{3} \mathrm{Si}>\delta-\mathrm{Ni}_{2} \mathrm{Si}>\theta-\mathrm{Ni}_{2} \mathrm{Si}>\alpha-\mathrm{NiSi}_{2}$. The volumetric thermal expansion coefficient $\alpha_{V}$, isochoric heat capacity $C_{v}$ and isobaric heat capacity $C_{p}$ of nickel silicides are proportional to $T^{3}$ at low temperature, subsequently, $\alpha_{V}$ and $C_{p}$ show modest linear change at high temperature, whereas $C_{v}$ obeys the Dulong-Petit limit. In addition, $\beta-\mathrm{Ni}_{3} \mathrm{Si}$ has the largest capability to store or release heat at high temperature. From the perspective of solid state physics, the thermodynamic properties at finite temperature can be used to guide further experimental works and design of novel nickel-silicon alloys.

DOI: 10.21883/FTT.2018.05.45875.292

* Полный текст статьи опубликован в журнале „Physics of the Solid State“. 